\title{
Assessing knowledge, attitude and practice of nurses after a continuing professional development program: A qualitative study
}

Jessie Johnson*1, Arlene Masaba ${ }^{1}$, Sadia Munir ${ }^{1}$, Robin O'Dwyer ${ }^{2}$, Amber Smith², Khalid Elawad ${ }^{2}$, Samya Ahmad Al Abdulla ${ }^{2}$

${ }^{1}$ Faculty of Nursing, University of Calgary, Qatar

${ }^{2}$ Primary Health Care Corporation, Qatar

Received: February 11, 2020

DOI: $10.5430 /$ ijh.v6n2p8
Accepted: March 23, 2020

Online Published: April 14, 2020

URL: https://doi.org/10.5430/ijh.v6n2p8

\begin{abstract}
Background: Nurses play a crucial role in the prevention of communicable diseases through the public health immunization programs. Knowledge, attitude and practices of health care providers have significant impact on the vaccine administration and education of adults and parents of children.

Objective: The present qualitative study aims to explore the knowledge, attitude and practices of nurses in Qatar before and after the delivery of the immunization-related continuous professional development program.

Methods: 10 out of 125 nurses who have completed the training program volunteered to participate in the face-to-face interviews. Data was then collected, transcribed and analysed by the researcher team members.

Results: Themes identified from this study: Empowerment, advancing practice and continuing education. Participants felt more confident and had a sense of fulfilment on completion of the education program. Practicing evidenced based skills and methods achieved an outcome of better quality of care, which can directly affect practice. Participants also felt that continuing education allows them to become self-motivated to keep up with new and emerging knowledge. Taken together, our data revealed that continuing professional education immunization-training program was successful in improving the knowledge, attitude and practices of the participants.

Conclusions: Continued education beyond traditional baccalaureate nursing programs in order to ensure nurses possess the knowledge to safely handle, teach and administer vaccines is needed.
\end{abstract}

Key Words: Immunizations, Vaccination, Nurses

\section{INTRODUCTION}

Nurses' knowledge, attitudes and practice can go a long way to successful uptake of vaccine administration. Nurses who work in the Primary Health Care Corporation (PHCC) in Qatar come from various parts of the world and possess varying levels of education related to the safe handling and administration of vaccines. Due to the nature of their work in primary health care, there is a need for nurses to be able to vaccinate various populations including children and adults, all of which come from various countries. To provide this service and to continue to encourage the population of Qatar to follow the guidelines for vaccination, nurses need to be

*Correspondence: Jessie Johnson; Email: jessie.johnson1 @ucalgary.edu.qa; Address: Faculty of Nursing, University of Calgary, Qatar. 
able to provide health education that enables them to have the ability to present themselves as confident health care practitioners. ${ }^{[1]}$ This is especially true when it comes to teaching and administration of vaccinations to adults and children. Nurses are perceived as trustworthy healthcare practitioners who are pivotal to encouraging vaccination compliance. Alhamaddi et al. ${ }^{[2]}$ suggests that confidence to provide accurate health information can stem from continued education and an understanding of how current best practice about the benefits of proper immunization allow nurses not only to care for clients and families. This change in knowledge, attitude and practice has an influence through continued education.

Vaccines were developed so humanity can find the means to eradicate communicable diseases. According to the World Health Organization, 3 million deaths occur worldwide caused by vaccine preventable disease. ${ }^{[3]}$ Though readily available, the uptake for vaccination worldwide has fallen to dangerous levels. ${ }^{[4]}$ Alhammadi, Khalifa, Abdulrahman, Almuslemani et al. ${ }^{[2]}$ stated that there is a pervasive hesitancy to vaccinate or recommend vaccination among healthcare professionals (HCP) in Qatar. This in part is due to lack of knowledge and varying cultural differences of the nursing workforce contained therein. ${ }^{[2]}$

It is of utmost importance that we investigate this topic because as we become global citizens, our travel and work enables us to traverse various areas of the world. Therefore, it is tantamount that nurses provide education and immunizations when available, to protect people against communicable diseases that are prevalent in the places where they live and work. Recent outbreaks and pandemics as well as former diseases eradicated in the past are emerging with serious consequences. ${ }^{[2]}$ Thus, nurses are poised to educate the population they serve to work toward a reduction in vaccine hesitancy and vaccine non-compliance. ${ }^{[4]}$ To do this, we need to explore knowledge, attitude and practice of nurses related to vaccine administration and education with adults and the parents of the children.

\section{METHOD}

This research utilized an interpretive description (ID) study design to explore nurses' knowledge, attitude and practice related to vaccinations before and after they participated in a professional development course. Interpretive description enables researchers the opportunity to understand the experience of participants by allowing questioning that is systematic in nature. ${ }^{[5]}$ In this design characteristics, patterns and structures can be identified by the researcher in order to construct knowledge about the phenomenon in question. ${ }^{[6]}$ The purpose of ID is to observe and recognize patterns and hence themes within the data in order to understand the is- sues with knowledge attitude and practice for nurses who work in PHCC. ${ }^{[7]}$ This method is beneficial for healthcare practitioners due to the useful applicability of the findings. ${ }^{[7]}$ Interpretive Description is well suited as a method for qualitative studies especially when the sample size is small and captures the realities of the subjective experiences such is the case in this study. ${ }^{[8]}$

\subsection{Ethics clearance}

Ethics obtained was from the research ethics board within Primary Healthcare Corporation prior to conducting this research. All data were kept in a computer that was password protected. The only person with access to the data was the lead principle investigator.

\subsection{Setting and sample}

This study was conducted with nurses who work in primary health care centers within Qatar. The participants had either a diploma or a bachelor's degree in nursing, worked in well baby clinics, school health clinics, chronic disease clinics and travel clinics. Participants were also required to have at least three years' experience working within the primary healthcare centres and had attended the three-day training related to vaccine, safe storage handling, education and administration. Once the training was complete, and after six weeks of practice with injections, participants became certified as vaccinator nurses. Once the LPI was notified of the certification, the responsible person in the work force training department within the primary healthcare centre sent an email apprising the participants of the research.

\subsection{Recruitment and data collection}

Participants were introduced to the research prior to commencing the continuing professional development vaccine program. The LPI informed them that after the program was complete and they were certified, they would be asked to volunteer to participate in face-to-face interviews lasting between 30 and 45 minutes in length. Questions included:

(1) Is there a particular case you found difficult to manage in your practice? If so, how did you manage it?

(2) What immunization topics do you feel are the most difficult to explain and how do you address this in your practice?

(3) What are the most significant myths you have heard about in your practice and how have you dealt with these in your practice?

(4) What tools would you find useful to have available to you in your practice to help in your understanding of immunizations when questions come up?

(5) What is your comfort level now that you have taken the 
immunization course when you begin to teach parents about the topic of immunization?

(6) What is your comfort level now that you have taken the immunization course in teaching adults about the necessity of getting and keeping their immunizations up to date?

One hundred and twenty five nurses completed the training program and signed of as certified vaccinators. However, the response rate was low after the program was completed thus, the researchers elected to take those that agreed to participate. Therefore, ten nurses were interviewed for the study. A transcriptionist who had signed a confidentiality consent transcribed audiotaped data verbatim. The four research team members reviewed each transcript, to verify and compared to each of the audio recording dates and times that matched to ensure accuracy.

\section{RESUlts}

\subsection{Data analysis}

It is important to note that when reporting in qualitative research it is crucial to recognize one's own bias in a process known as reflexivity prior to and when analyzing the data. Interpretive description consists of synchronous data collection and analysis as one informs the other. ${ }^{[8]}$ Three of the researchers who participated in this study analyzed transcribed audiotapes. Thematic analysis took place as each researcher listened to the audio recordings and then read the transcripts to become familiar with the content. Once complete they came together to discuss any differences they may have had and discuss congruence of themes. After analysis, three themes emerged from the data; these themes were Empowerment, Advancing Practice and Continuing Education.

\subsection{Themes}

\subsubsection{Empowerment}

Defined as the process of becoming stronger and more confident, especially in controlling one's life and claiming one's rights. ${ }^{[9]}$ In order for nurses to practice effectively they need to feel empowered enough to take on the role of vaccination nurse. Nurses felt that participating in the course empowered them with new knowledge directly relatable to their practice. This provided them with the confidence to collaborate with parents in cases where they otherwise would not have. In addition, prior to undertaking the program, many nurses were afraid to ask or answer questions, fearing they might not know the answer to parents' questions. Some thoughts relating to this are evidenced in the below quotes.

Before, I had lack of confidence. It stopped me from educating parents. I changed. (N3)
I used to be afraid but now I have a good idea about vaccines, I can give information. If I didn't know I would not answer. Now I am waiting for their questions. (N2)

The nurses felt they had a sense of fulfilment and belonging to the team armed with this new knowledge from the education program, they attended. They also felt a sense of empowerment they say never existed before they participated in the training.

I have experience now and I feel good, I did well with practice. (N6)

Before, I had lack of confidence. It stopped me from educating parents. I changed. (N2)

Overall, the training provided nurses with the tools they need to be able to feel comfortable speaking with patients about information or issues surrounding vaccination compliance and uptake which had an impact on their practice as they worked to become proficient within their respective clinics.

\subsubsection{Advancing practice}

Participating in the vaccine training was necessary in order to advance nursing practice. Nurses who participated felt they were able to dispel myths parents held relating to the efficacy and dangers of vaccination. Overwhelmingly many participants were proud of their new ability to explain information related to immunizations, herd immunity and understand defaulters (those that do not follow through with continued vaccines) with ease $N 1,3,4,7,8,9,10$. Nurses are the most trusted profession and having this training advanced their skills and abilities in the area of vaccinations. Many participants state they now spends more time providing education and care about vaccination. Some went on to say that:

This had a chain reaction in the attitudes and behaviours of parents, in particular trust in the nurse and anxiety and cooperation. (N10)

If time taken to explain more, they have trust [in us] and [tell us] their concerns. This make them more comfortable, make them in a comfort zone ... Taking the time to explain in other terms, other ways... this reduce[s] parent anxiety... (N4)

Parents feel good.....we give lots of information... (N7)

Practicing evidenced based skills leads to better quality of care, which can directly impact and advance practice. By increasing the quality care parents witnessed, the impact as noted by one of the participants below.

ISSN 2377-7338 E-ISSN 2377-7346 
In the past we were forcefully giving vaccinations on the bed, but now once parents come to know the experience, they will realize that sitting holding the child is better. (N1)

Parents come back for the next visit and because of improved service of giving immunization there are less defaulters as a result....parents returning for vaccination. (N4)

The impact of the training is evidenced by the fact nurses have the knowledge to inform parents of evidence related to practice, are able to give instructions with rationale, and spend time to get informed consent to communicate about vaccinations etc. This in turn has enabled parents to be more confident with the nurses' skills.

\subsubsection{Continuing education}

Overall, nurses felt the need to maintain their education in order to effect care. Continuing education allows nurses to be abreast of any new and emerging knowledge that may present itself. It also continues to enhance their profile and thus provides parents and patients with the reality that nurses are versed in the care they enact. This is evidenced by the quote below.

I want to continue in vaccination... good for explanation. (N9)

We didn't have a good idea about vaccinations but now we do. (N10)

If you will not refresh, you will forget; the practising is different from what is in your mind. (N1)

Having this education provided nurses with the selfmotivation to strive to learn. They demonstrated an energy and spirit of creativity to improve resources and to create resources for parents and adults.

We use pictures to teach Arabic patients when we don't speak the language. (N8)

We make posters so that parents can see different things related to vaccines. (N2)

Many participants wanted continuing professional development refresher courses in the future. They stated that it would be beneficial as some nurses do not give vaccines regularly and are afraid they will forget what they had previously learned. They felt the need to keep abreast of the information from sites such as the WHO or CDC. Continuing education is tantamount to effecting good practice.

\section{Discussion}

Tantamount to the successful education and compliance of adults, and parents of children requiring immunizations, is the need for continuing professional development programs for healthcare workers, especially nurses. Nurses are at the forefront of successful campaigns to increase vaccine compliance and are pivotal to the successful uptake of vaccination rates in healthcare today. They are the frontline ambassadors to dispelling myths and negative beliefs about how parents think and feel about receiving vaccines. ${ }^{[13]}$ Thus, it is important to deliver education that empowers, advances practice and is continuing in its nature.

\subsection{Empowerment}

Nurses provided specialized education to lead evidence based practice initiatives are more apt to do so if they feel empowered. ${ }^{[10]}$ Nurses in the present study felt empowered by the education they received related to vaccines. Enabling nurses to avail of specialized education related to vaccines is the gateway to empowering them to create change. ${ }^{[11]}$ It also goes a long way to strengthening the organization within which they work. ${ }^{[10]}$ In a qualitative study conducted by Chaghari et al., ${ }^{[12]}$ which looked at the viewpoints and experiences of 35 nurses to in-service education, they found that empowering education has an influence on the practice of participants and led to self-directed learning. They further state that mandated education that does not empower practice leads to poor compliance and uptake, whilst education that empowers practice lead to more successful outcomes for healthcare delivery thus advancing practice.

\subsection{Impacting practice}

As nurses in this study come from various countries and possess varying degrees of education when it comes to vaccine knowledge and practice, a continuing professional development program such as this was imperative. Nurses readily expressed the fact this program advanced their practice. In addition, being equipped with the information needed to work in vaccine clinics led to more successful uptake of immunizations. Nurses need to be up to date and are pivotal to the success of vaccine campaigns worldwide. Roles have expanded to include initiatives related to public health campaigns, and an advanced knowledge of communicable diseases. ${ }^{[13]}$ An integrative review conducted by Fagundas et al., ${ }^{[14]}$ which looked at nursing practices related to vaccines. They found that the role of the nurse is a key element in pulling together interprofessional teams to contribute to primary goals of primary healthcare. ${ }^{[14]}$ They also found that practice was "impacted through continuing education of nursing staff to plan strategies to reach the goals of vaccination" (p. 128). Thus, it is necessary to provide continuing 
education to nurses as well as other healthcare clinicians so that practices remain relevant and up to date.

\subsection{Continuing education}

Knowledge related to the safe handling and administration of vaccines is essential to ensure success and enhance uptake. Nurses in this study felt they needed extra knowledge over and above what is provided in school, in order to feel comfortable in administering vaccine information to adolescents, adults and parents. They also felt that offered continuing education as inextricably linked to the successful uptake of vaccines going forward. In a study conducted by Chan et al., ${ }^{[15]}$ which looked at having a nurse led education campaign for pneumococcal vaccines, results demonstrated that nurses who provided the education and training were empowered with new knowledge and hence uptake rates of vaccines increased twofold. This was also echoed in Werk, 2019 ${ }^{[16]}$ study which looked at web based training as a way of continuing education for clinicians to promote the uptake of seasonal influenza vaccine. This study demonstrated that the use of continuing education for clinicians had a positive effect on the uptake of the vaccine by $55.8 \%$ for children and adolescents. Continuing education provides a way of keeping clinicians current with vaccine schedules and everyday practice related to the administration and knowledge related to vaccines. It can also go a long way to increasing patient satisfaction and cost effectiveness of healthcare service utilization from diseases that can be avoided once immunized. ${ }^{[17]}$ Providing the opportunity for continuing education for clinicians is tantamount to the success in vaccination campaigns both locally and globally.

\section{Conclusion}

As previously, stated Nurses were seen as trustworthy healthcare practitioners and their role in encouraging vaccination compliance is undisputed, if we as a profession are to support the eradication of communicable diseases by means of vaccinations then we all must play a part in keeping up to date with knowledge and skills related to vaccines/immunizations.

PHCC in Qatar recognized this gap in knowledge and requested support for a program specifically for frontline nurses. This purposely-formulated development program considered these requirements and further included the nurses' ethnicity, level of education and experience; and thus identified as being important considering the multicultural workforce and population in Qatar.

The knowledge attitudes and practices observed during this continuing professional development program highlight a deeper level of professional growth, in knowledge, understanding, education and skill thus creating a shift within the participants from a lack of confidence in this area, to an empowered nurse able to educate, support and lead.

The success of this continuing professional development program attributed to localizing the delivery of course to meet the needs of the multicultural workforce. The impact to practice has been a ripple effect where the newly empowered nurses are no longer afraid to answer questions of patients and now leading vaccination clinics as a resource and or mentors. Future development of regular updates and an interprofessional vaccination training course has been considered to include physicians, pharmacy and nurses in order to further cement the professional obligation to support the eradication of communicable disease thru empowered, confident health professionals.

\section{Conflicts of InTERest Disclosure}

The authors have no conflicts of interest to declare and have contributed equally to this manuscript.

\section{REFERENCES}

[1] Grinspun D, Bajnok I. Transforming Nursing Through Knowledge. Sigma Theta Tau. 2018.

[2] Alhammadi A, Khalifa M, Abdulrahman H, et al. Attitudes and perceptions among the pediatric health care providers toward influenza vaccination in Qatar: A cross-sectional study. Vaccine. 2015; 33(32): 3821-3828. PMid:26144904. https://doi.org/10.1016/j.va ccine.2015.06.082

[3] World Health Organization. WHO vaccine-preventable disease: Monitoring system. 2017 gobal summary. 2018. Available from: http://apps.who.int/immunization_monitoring/g lobalsummary/coverages?c=QAT

[4] Butler R, MacDonald NE. Diagnosing the determinants of vaccine hesitancy in specific subgroups: The Guide to Tailoring Im- munization Programmes (TIP). Vaccine. 2015; 33(34): 4176-4179. PMid:25896376. https://doi.org/10.1016/j.vaccine. 2015 .04 .038

[5] Thorne SE. Interpretive Description. Walnut Creek, CA: Left Coast Press; 2008.

[6] Thorne S, Reimer Kirkham S, MacDonald-Emes J. Interpretive description: a non-categorical qualitative alternative for developing nursing knowledge. Research and Nursing Health. 1997; 20(6): 169-177. https ://doi .org/10.1002/(SICI) 1098-240X (1997 04) $20: 2<169:$ :AID-NUR9>3.0.CO;2-I

[7] St George S. Applied Interpretation: A review of interpretive description by Sally Thorne. The Qualitative Report. 2010; 15(6): 16241628.

[8] Thorne S, Kirkham SR, O'Flynn-Magee K. The analytic challenge in 
interpretive description. International Journal of Qualitative Methods. 2004; 3: 1-21. https://doi.org/10.1177/1609406904003001 01

[9] Empowerment. In Oxford Online Dictionary. 2019. Available from: https://en.oxforddictionaries.com/definition/e mpowerment

[10] Melnyk BM, Fineout-Overholt E, Gallagher-Ford L, et al. The state of evidence-based practice in US nurses: Critical implications for nurse leaders and educators. The Journal of Nursing Administration. 2012; 42(9): 410-417. PMid:22922750. https://doi.org/10.1 097/NNA . Ob013e3182664e0a

[11] Melnyk BM, Gallagher-Ford L, Long LE, et al. The establishment of evidence-based practice competencies for practicing registered nurses and advanced practice nurses in real-world clinical settings: Proficiencies to improve healthcare quality, reliability, patient outcomes, and costs. Worldviews on Evidence-Based Nursing. 2014; 11(1): 5-15. PMid:24447399. https://doi.org/10.1111/wvn.12021

[12] Chaghari M, Ebadi A, Ameroyun A, et al. An attempt for empowering education: A qualitative study of in-service training of nursing personnel. Iranian Journal of Nursing and Midwifery Research. 2016; 21(15): 498-503. PMid:27904634. https://doi.org/10.4103/ $1735-9066.193404$
[13] Frenk J, Chen L, Bhutta Z, et al. Health professionals for a new century: transforming education to strengthen healthcare systems in an interdependent world. Lancet. 2010; 376(9756): 1923-158. https://doi .org/10.1016/S0140-6736(10)61854-5

[14] Fagundas L, Frota O, Silva E. Nursing practices in vaccination: An integrative review. Journal of Nursing Education and Practice. 2018; 8(8): 128-136. https://doi.org/10.5430/jnep.v8n8p128

[15] Chan SC, Leung DYP, Leung AYM, et al. A nurse-delivered brief health education intervention to improve pneumococcal vaccination rate among older patients with chronic diseases: A cluster randomized controlled trial. International Journal of Nursing Studies. 2014; 52(1): 317-324. PMid:25012957. https ://doi .org/10.1016/j . ijnurstu.2014.06.008

[16] Mash R, Kroukamp R, Gaziano T, et al. Patient education costeffectiveness of a diabetes group education program delivered by health promoters with a guiding style in underserved communities in Cape Town, South Africa. Patient Education and Counseling. 2015; 98: 622-626. PMid:25641665. https://doi.org/10.1016/j.pe c. 2015.01 .005

[17] National League for Nursing. Mission/goals/core values. [Cited 3 Feb 2015.] 2013. Available from: http://www.nln.org/aboutn ln/ourmission.htm 